\title{
Las habilidades básicas del pensamiento en tutorados normalistas
}

\section{Basic Thinking Skills in Tutorial Normalistas}

DOI: $10.46932 /$ sfjdv3n1-089

Received in: Jan 30st, 2021

Accepted in: Feb 1th, 2022

\author{
Carlos Alfredo Damián García \\ Dr. En Investigación Educativa \\ Esc. Normal Experimental \\ "Salvador Varela Reséndiz" \\ Av.de la Normal s/n Juchipila, Zac. México \\ E-mail: cabritoreposado@gmail.com
}

\section{RESUMEN}

El presente trabajo de investigación tuvo el objetivo de verificar si los procesos básicos del pensamiento mejoran después de la aplicación del taller Habilidades del Pensamiento. Se realizó con 2 grupos del segundo semestre de la Escuela Normal Experimental "Salvador Varela Reséndiz" de Juchipila, Zacatecas: grupo 1 de la Licenciatura en Educación Primaria y grupo 2 de la Licenciatura en Educación Preescolar. Fue un estudio experimental sin grupo control, donde se aplicó un pre-test permitiendo conocer el estado inicial de los procesos básicos de pensamiento de los estudiantes. Posteriormente, se les impartió un taller para desarrollar el conjunto de habilidades del pensamiento lógico tomando en cuenta las diferentes operaciones y esquemas mentales del conocimiento, basadas en la propuesta de Sánchez, M. (2002). Por último, se aplicó un post-test, dando resultados de un aumento en los procesos básicos del pensamiento (observación, clasificación y seriación) que a su vez influyen en la solución eficaz de matrices analógicas y solución de problemas lógicos.

Palabras clave: procesos básicos del pensamiento, desarrollo cognitivo, tutorías, formación docente.

\begin{abstract}
The present research work had the objective of verifying if the basic processes of thought improve after the application of the Thinking Skills workshop. It was carried out with 2 groups from the second semester of the "Salvador Varela Reséndiz" Experimental Normal School in Juchipila, Zacatecas: group 1 of the Bachelor of Primary Education and group 2 of the Bachelor of Preschool Education. It was an experimental study without a control group, where a pre-test was applied, allowing to know the initial state of the basic thought processes of the students. Subsequently, they were given a workshop to develop the set of logical thinking skills taking into account the different operations and mental schemes of knowledge, based on the proposal of Sánchez, M. (2002). Finally, a post-test was applied, giving results of an increase in the basic processes of thought (observation, classification, and seriation), which in turn influence the effective solution of analog matrices and the solution of logical problems.
\end{abstract}

Keywords: basic thought processes, cognitive development, tutorials, teacher training. 


\section{INTRODUCCIÓN}

Las habilidades básicas de pensamiento son procesos mentales que permiten el manejo y la transformación de la información, facilitan la organización y reorganización de la percepción y la experiencia. Por medio de dichos procesos se comprende, se reflexiona, se analiza, se argumenta, se crean y recrean realidades, se construyen y reconstruyen significados. Según Sánchez (2002), dichos procesos se pueden desarrollar y perfeccionar con la práctica, y hasta convertirlos en habilidades ya que el proceso existe por sí mismo, independientemente de la persona que lo ejecuta, elprocedimiento proviene de la operacionalización del proceso y la habilidad es una facultad de la persona, cuyo desarrollo requiere de un aprendizaje sistemático y deliberado.

La coordinación del área de tutorías de la Escuela Normal Experimental "Salvador Varela Reséndiz" identificó conbase en los resultados de los exámenes de admisión, test de hábitos de estudio y el análisis de las trayectorias escolares, que había una problemática con los procesos mentales que permiten el manejo y trasformación de información.

ANUIES (2000) dice "la tutoría es un acompañamiento personal y académico a lo largo del proceso formativo paramejorar el rendimiento académico, facilitar que el estudiante solucione sus problemas escolares, desarrolle hábitos deestudio, trabajo, reflexión y convivencia social". Dentro de uno de los 3 programas que constituyen la tutoría en ésta escuela, referente a la atención a estudiantes de nuevo ingreso (dirigido a alumnos de primer a segundo semestre), se decidió implementar un taller para desarrollar las habilidades básicas del pensamiento y así dar atención a esta problemática identificada.

Los alumnos de $7^{\circ}$ semestre de la Lic. en Psicología Educativa del campus Jalpa de la Universidad Autónoma deZacatecas, aplicaron un taller basado en el paradigma de procesos para el desarrollo de habilidades del pensamiento de Margarita Amestoy de Sánchez (2002), el cual consistió en el desarrollo de las habilidades básicas del pensamiento(observación, clasificación y seriación) que a su vez influyeron en la solución eficaz de matrices analógicas y soluciónde problemas lógicos; esto fue con la finalidad de que los alumnos de $2^{\circ}$ semestre de la Escuela Normal cuenten con herramientas que les permitan hacer procesos mentales efectivos y beneficiosos para ellos, que influya en una trayectoria escolar exitosa. El objetivo del presente trabajo fue verificar si los procesos básicos del pensamiento mejoran después de la aplicación del taller.

De acuerdo a la SEP (2018), el modelo educativo define la formación docente como "la preparación para el ejercicio docente. Comprende el desarrollo de saberes relacionados con los contenidos de la enseñanza, así como los conocimientos y habilidades en términos pedagógicos que se requieren en dicha tarea" (, p. 208). Por lo tanto, esta investigación puede repercutir positivamente en el desarrollo de las competencias genéricas de los estudiantes normalistas, concretamente, en la capacidad para 
aprender a aprender por iniciativa e interés propio a lo largo de lavida y la capacidad para solucionar problemas a partir de métodos establecidos.

\section{MÉTODO}

El presente estudio es un experimento sin grupo control, donde participaron 2 grupos: el grupo 1 estuvo integrado por 24 alumnos de la Licenciatura en Educación Primaria y el grupo 2 estuvo conformado por 28 alumnos de la Licenciatura en Educación Preescolar.

Primero, se aplicó el pre-test de desarrollo de habilidades del pensamiento validado por Corona L. y Vital A. (2014),(se encuentra en apéndice). Enseguida, se realizó un taller que siguió una secuencia de pasos o procesos para que se desarrollara el pensamiento, según el paradigma de Amestoy de Sánchez (2012):
1. Observación.
2. Comparación.
3. Relación.
4. Clasificación.
5. Ordenamiento.
6. Clasificación jerárquica.
7. Análisis y síntesis.
8. Evaluación.

Este paradigma indica que las habilidades básicas del pensamiento son aquellos procesos mentales que utilizamos en la vida diaria para resolver problemas que se presentan y ayudan a organizar y reorganizar la información que percibimos según sea necesario. Dicho taller tuvo una duración de 5 sesiones de 2 horas cada una. Al terminar la intervención, se aplicó el mismo test, pero en calidad de post-test.

\section{RESULTADOS}

El análisis de datos se realizó a través del programa estadístico SPSS V.20, donde se identificó, al comparar las medias entre pre- test- post-test de ambos grupos, diferencias mínimas en los promedios generales de los procesos evaluados entre el pre-test y post-test que no resultaron significativas estadísticamente.

En la tabla 1, se muestran los resultados obtenidos en la aplicación del pre-test, así como los resultados del post-test de los 2 grupos de $2^{\circ}$ semestre de la Escuela Normal de Juchipila, Zac. 
Tabla 1. Comparación entre los resultados obtenidos del Pre-test y el Pos-test de manera grupal.

\begin{tabular}{ccccc}
\hline $\begin{array}{c}\text { Grupo/ } \\
\text { Licenciatura }\end{array}$ & No. De Alumnos & Promedio Pre-Test Promedio Post-Test & $\neq$ \\
\hline 1. Primaria & 24 & $50.21 \%$ & $53.3 \%$ & 3.09 \\
2. Preescolar & 28 & $48.7 \%$ & $57.5 \%$ & 8.8 \\
\hline
\end{tabular}

Como se puede verificar en los datos, el grupo de Preescolar obtuvo una mayor diferencia de puntajes entre elpre- test y el post-test.

La figura 1 muestra los resultados por proceso del grupo 1 de la Lic. en Educación primaria.

Figura 1. Gráfico comparativo de promedios obtenidos por procesos entre los resultados obtenidos del Pre-test y el Pos-test del grupo 1 de la Licenciatura en Educación Primaria.

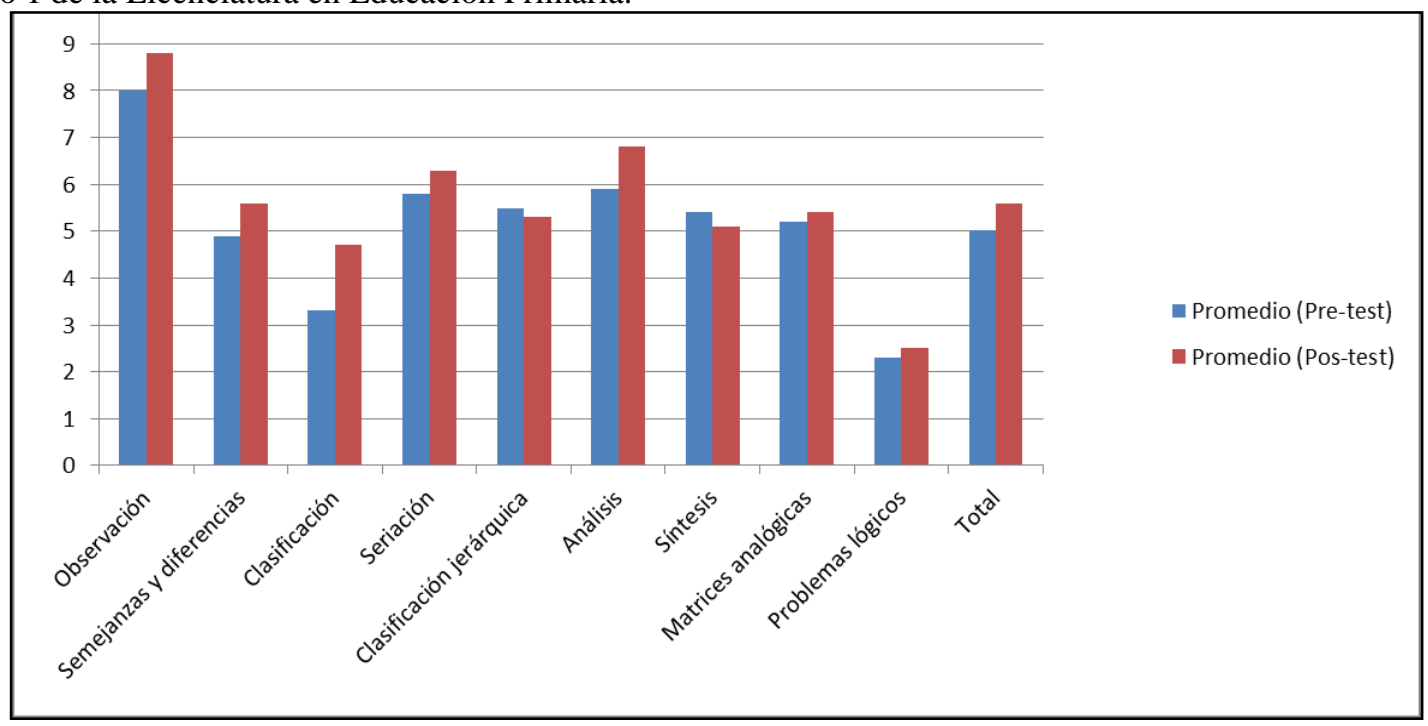

Como se puede visualizar, el proceso de observación es el que tuvo el mayor puntaje y mejor diferencia entre elpre-test y pos-test. En cambio, el de problemas lógicos es el de menor puntaje y menor diferencia.

La figura 2 muestra los resultados por proceso del grupo 2 de la Licenciatura en Educación Preescolar. 
Figura 2. Gráfico comparativo de promedios obtenidos por procesos entre los resultados obtenidos del Pre-test y el Pos-test delgrupo 2 de la Licenciatura en Educación Preescolar.

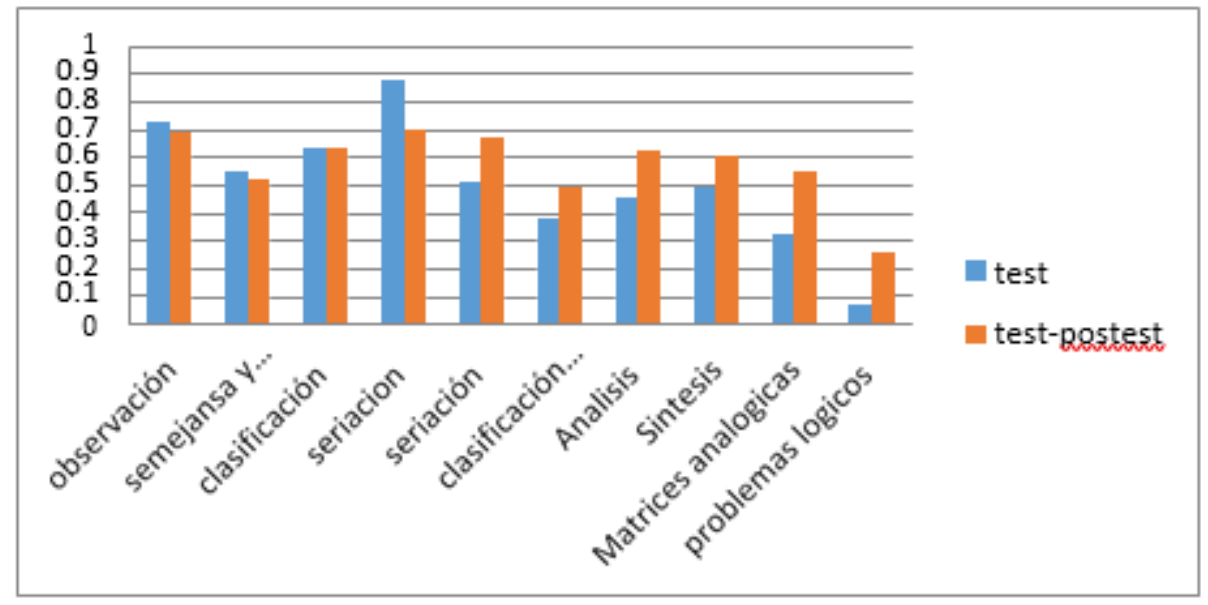

Como se puede observar en el grupo de Lic. en Educación Preescolar, los primeros 4 procesos (observación, semejanzas, clasificación, seriación) se realizaron mejor en el pre-test y en los últimos 6 procesos se identifica un avance en puntuación en el post-test.

\section{CONCLUSIONES}

Al aplicar el taller para desarrollar las habilidades básicas del pensamiento, bajo el paradigma de procesos de Amestoy de Sánchez (2002), se pudo observar las siguientes conclusiones. Hubo mejoría en cuanto al desarrollo de las habilidades del pensamiento de manera individual, demostrado por la diferencia de los promedios individuales, enla mayoría de los casos. Dicha diferencia se ratificó con la evaluación verbal que se hizo al final del taller, por parte de los alumnos de la Escuela Normal de Juchipila. Como lo cita (Olea y Salazar 2021 de ANUIES 2011, P.38) "La tarea del tutor, entonces, consiste en estimular las capacidades y procesos de pensamiento, de toma de decisiones y de resolución de problemas".

También, aunque las diferencias entre post-test y pre-test no fueron significativas estadísticamente de manera grupal, al hacer un análisis detallado por proceso y por persona, se encuentran datos interesantes. Unos datos validan los planteamientos teóricos del desarrollo cognitivo, tal como se puede ver en la figura 1 del grupo de primaria, donde lamayor puntación está reportada en los procesos más generales como observación, y al llegar a los problemas lógicos, se identifica una baja en los puntajes obtenidos, por la misma complejidad e integración de los procesos de análisis, síntesis y evaluación.

Hay otros datos que llaman la atención y habrá que realizar un análisis pormenorizado, comparando este estudio con otros datos que se tienen sobre el grupo de preescolar, ya que la mayor puntación de procesos está en seriación, no en observación como se esperaría con base al sustento teórico del taller. 
Así mismo, se observó que hay una baja de puntaje entre pre-test y post-test, ya que el primero fue mayor que el segundo. Lo anterior muestra que es necesario un análisis minucioso por proceso y por persona, integrando otras variables como el puntaje en la prueba de admisión y test de hábitos de estudio; Esto podrá permitir tener una evidencia certera del desarrollo cognitivo de cada alumno, para integrarlo al expediente de tutorías, atender las fortalezas y áreas de mejora de cada alumno, posibilitando una trayectoria académica exitosa. 


\section{REFERENCIAS}

ANUIES, (2001) La Educación Superior en el Siglo XXI; Líneas estratégicas de desarrollo, Una propuesta de ANUIES.

Corona L., Vital A. (2014) Estudio sobre el Desarrollo de Habilidades básicas del pensamiento en alumnos de $6^{\circ}$ semestre de Bachillerato. Memorias en extenso del 16 verano de la Ciencia. Región centro.

ISBN

978-607-441-302-1

http://www.veranos.ugto.mx/memorias/16VeranoCienciaRegionCentro/VRC_2014_Memorias.pdf

Olea, E. y Salazar, R. (2021). La acción tutorial en el posgrado: Reflexiones y principales desafíos en la formación investigativa. https://southfloridapublishing.com/ojs/index.php/jdev/article/view/725/658

Sánchez, M. (2002). La investigación sobre el desarrollo y la enseñanza de las habilidades de pensamiento. Revista Electrónica de Investigación Educativa 4, (1). En http://redie.uabc.mx/vol4no1/contenidoamestoy.html

Sánchez, M. (2012). Procesos básicos del pensamiento. México: Trillas.

SEP,

(2018).

Modelo

Educativo.

En

http://media.educacioncampeche.gob.mx/file/file_a36334cd9aebe41e1197b4a403da9741.pdf 


\section{APÉNDICE}

TEST PARA MEDIR EL DESARROLLO DE LAS HABILIDADES BÁSICAS DEL PENSAMIENTO VALIDADO POR CORONA L. Y VITAL A. (2014)UTILIZADO EN LA INVESTIGACIÓN
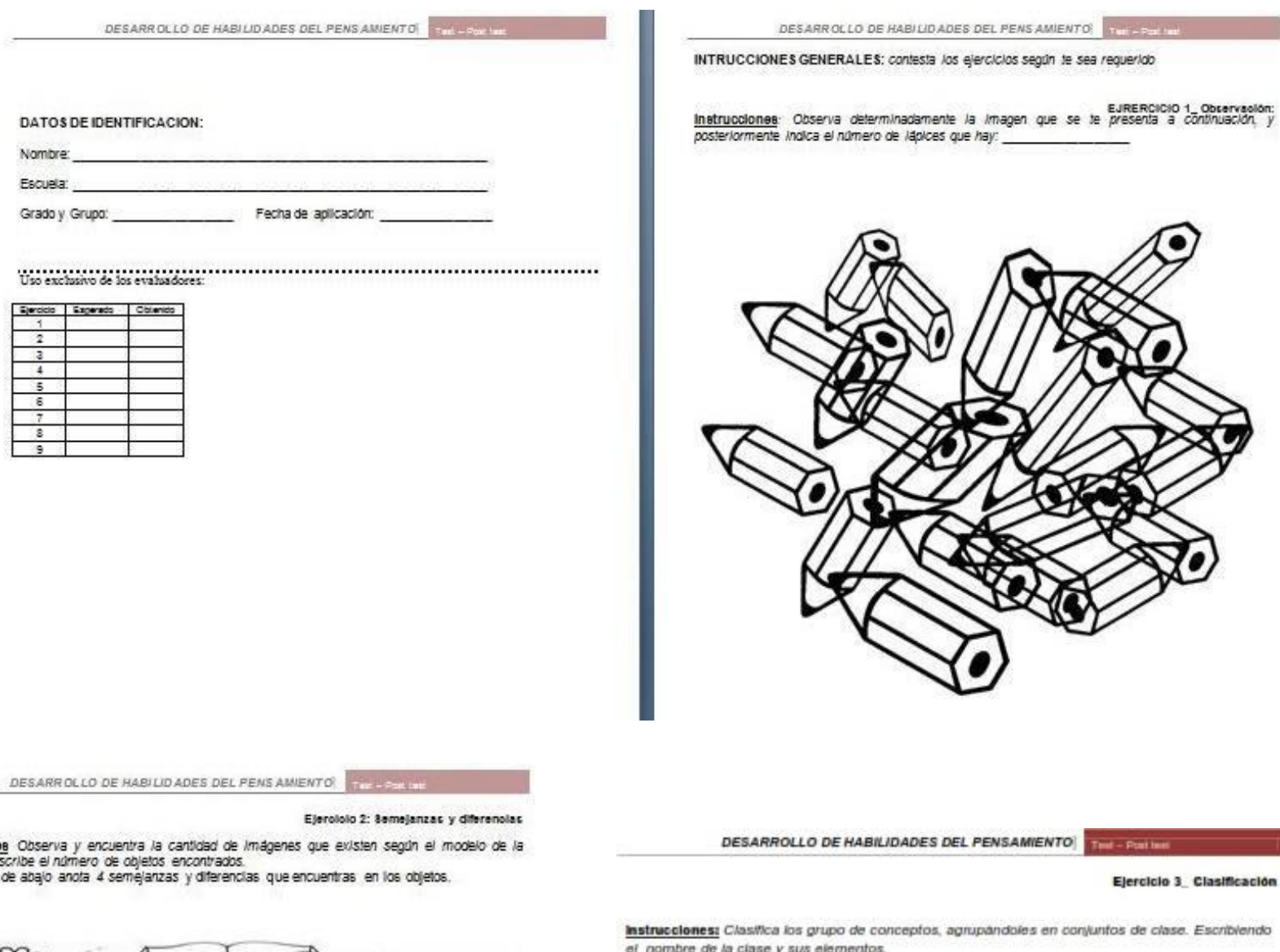
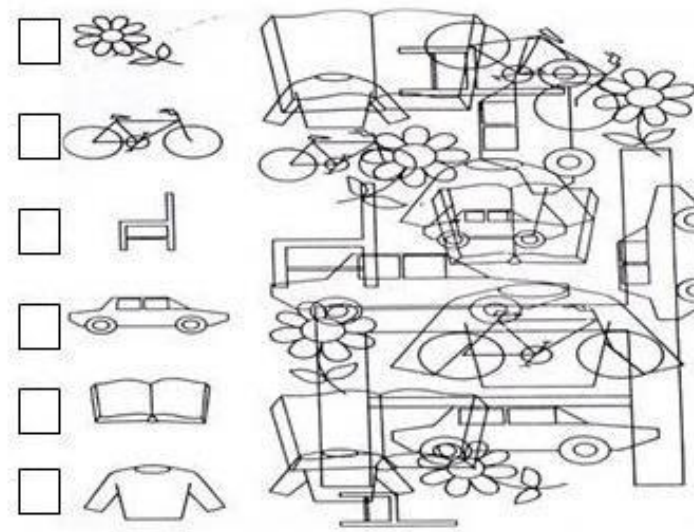

DATOS DE IDENTIFICACION:

Escuses:

Graso y Grups:

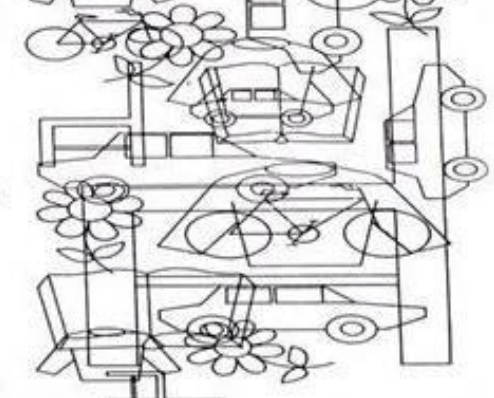

\begin{tabular}{|c|c|c|c|c|}
\hline & $\begin{array}{l}\text { ODIJOA } \\
\text { MEMEJANTEa }\end{array}$ & $\begin{array}{l}\text { PPor que con } \\
\text { camajantac? }\end{array}$ & $\begin{array}{c}\text { Ob/jhoc } \\
\text { DIFERENTE }\end{array}$ & $\begin{array}{l}\text { 2Por que con } \\
\text { ollarontace? }\end{array}$ \\
\hline \multicolumn{5}{|l|}{$\frac{1}{2}$} \\
\hline 3 & & & & \\
\hline & & & & \\
\hline
\end{tabular}


Fiercicio 4. Seriacibon Instrucciones: tee bo ajowente

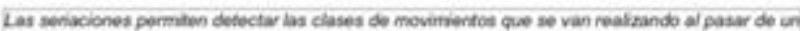
estado a oto

Tipes de series.

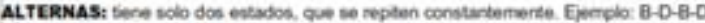

CiCLICAS: Senen mas de dos estados y vuelven diclicamente el primero, pero sin pasar por los

intermedios. Epemblo. A-B-C-A.O-C.

PENDULARES: tenen más do dos estados y voelven al pertera, pero pasando otra vez por los

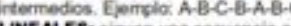

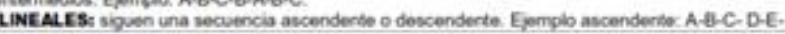

Instruscienes Complua las series de hibas colbcando las que den continuidad a la sesie y

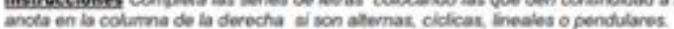

completa La Baive

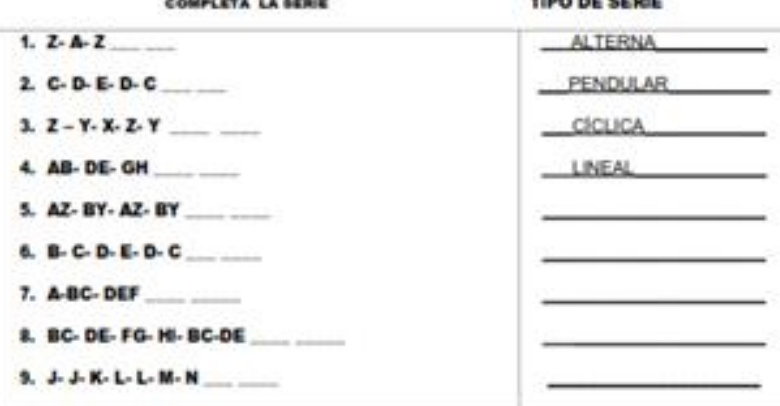

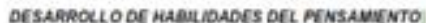

Epercikio 5 Seriacien 2

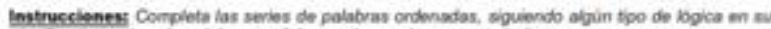

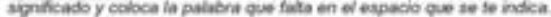

1. Charce- entanque - lage - mar

Enconder-mogur-encender .

Mudo - calads - hablador

Raiz - bonce - ramas

hores

5. Amanecer

6. Pómo

malo - rogular - bueno - mepor

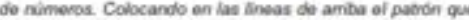
se sigue y en la parte de abayo los numboros que completen la nerie.

Ejemplo:
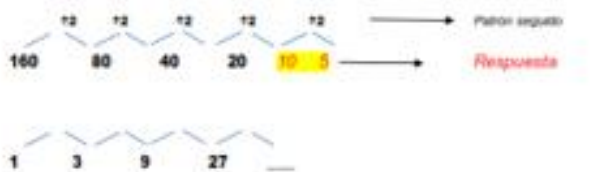

(1) 14 24 22

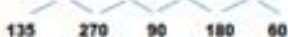

Instrucciones Realiza una clasificación de las siguientes fouras fornando en cuento la jerarquia de clases y sub clases foma en cuenta las siguientes variables: Forma, tamahlo, y la intensidad do color. Ancta los numeros do cada una de las clases.

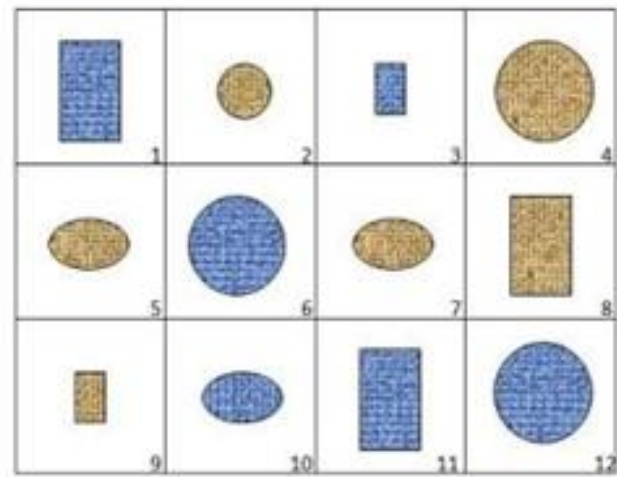

DESARROLLO DE HABILIDADES DEL PENSAMIENTO

Test-pontert

Ejercicio 7 Analisis

Instrucciones: Lee la siguiente información y realiza lo que se te solicila

LA EMPRESA COREANA TENE UN GERENTE GENERAL, EL CUML OPERA CON DOS GERENTES DE ÄEEA, CADA UNO, A SU VEZ CONTROLA DOS JEFES DE DEPARTAMENTO, OUIENES CRCANIZAN SUS GRUPOS DE PERSONRL AOMINISTRATIVO Y OERERO. ANRLIZA LA ESTRUCTURA DE LA ORGANIZACION DE ESTA EMPRESA Y REPRESENTALA MEOUNTE UN DUAGRAMA. 
